OPEN ACCESS

Edited by:

Zhe-Sheng Chen,

St. John's University, United States

Reviewed by:

Khushboo Jani,

RAPT Therapeutics, United States

Yiyi Liu,

Guangzhou Medical University, China

*Correspondence:

Jing $L$

lijing7405@126.com

Ping Liu

Ipivy@126.com

Chunlin Chen

jieru@163.com

${ }^{\dagger}$ These authors share first authorship

Specialty section: This article was submitted to

Molecular Diagnostics and

Therapeutics,

a section of the journal

Frontiers in Molecular Biosciences

Received: 29 September 2021 Accepted: 06 December 2021 Published: 09 February 2022

Citation:

Liu L, Lv J, Lin Z, Ning Y, Li J, Liu P and Chen $C$ (2022) Co-Overexpression of GRK5/ACTC1 Correlates With the

Clinical Parameters and Poor Prognosis of Epithelial Ovarian Cancer.

Front. Mol. Biosci. 8:785922.

doi: $10.3389 /$ fmolb.2021.785922

\section{Co-Overexpression of GRK5/ACTC1 Correlates With the Clinical Parameters and Poor Prognosis of Epithelial Ovarian Cancer}

\author{
Longyang Liu ${ }^{1,2 \dagger}$, Jin $\mathrm{Lv}^{2,3+}$, Zhongqiu Lin ${ }^{4}$, Yingxia Ning ${ }^{5}$, Jing $\mathrm{Li}^{1 *}$, Ping Liu ${ }^{1 *}$ and \\ Chunlin Chen $^{1 *}$
}

${ }^{1}$ Department of Gynecology and Obstetrics, Nanfang Hospital, Southern Medical University, Guangzhou, China, ${ }^{2}$ Cancer Center, Integrated Hospital of Traditional Chinese Medicine, Southern Medical University, Guangzhou, China, ${ }^{3}$ Department of Obstetrics and Gynecology, Longgang Central Hospital of Shenzhen City, Shenzhen, China, ${ }^{4}$ Department of Gynecology Oncology, The Memorial Hospital of Sun Yat-sen University, Guangzhou, China, ${ }^{5}$ Department of Gynecology and Obstetrics, The First Affiliated Hospital of Guangzhou Medical University, Guangzhou, China

Background: The prognosis of epithelial ovarian cancer (EOC) is poor, and the present prognostic predictors of EOC are neither sensitive nor specific.

Objective: The aim of this study was to search the prognostic biomarkers of EOC and to investigate the expression of G protein-coupled receptor kinase 5 (GRK5) and actin alpha cardiac muscle 1 (ACTC1) in EOC tissues (both paraffin-embedded and fresh-frozen tissues) and to explore their association with clinicopathological parameters and prognostic value in patients with EOC.

Methods: A total of 172 paraffin-embedded cancer tissues of EOC patients diagnosed and operated at the memorial hospital of Sun Yat-sen University between December 2009 and March 2017 and 41 paratumor tissues were collected and the expression of GRK5 and ACTC1 was examined using immunohistochemistry. Furthermore, 16 fresh-frozen EOC tissues and their matched paratumor tissues were collected from the Integrated Hospital of Traditional Chinese Medicine, Southern Medical University, between August 2013 and November 2019 and subjected to reverse-transcription quantitative PCR analysis to detect the mRNA expression of GRK5 and ACTC1.

Results: The expression of GRK5 and ACTC1 was both higher in cancer tissues than in paratumor tissues. GRK5 expression was positively correlated with ACTC1 expression. In addition, GRK5, ACTC1, and GRK5/ACTC1 expression was associated with the recurrence-free survival and overall survival of EOC patients. Furthermore, multivariate logistic regression analysis indicated that GRK5+/ACTC1+ co-expression, intestinal metastasis, postoperative chemotherapy, platinum resistance, and hyperthermic intraperitoneal chemotherapy were independent prognostic factors of EOC.

Conclusion: GRK5 and ACTC1 are both upregulated in EOC compared with those in paratumor tissues. The co-expression of GRK5+/ACTC1+ rather than GRK5 or ACTC1 is an independent prognostic biomarker of EOC.

Keywords: GRK5, ACTC1, epithelial ovarian cancer, prognosis, expression 


\section{INTRODUCTION}

Ovarian cancer (OC) is the main cause of mortality in female reproductive malignant cancers in China (Chen et al., 2016), and it is the second most common cause of gynecologic cancer-related death in women worldwide (Lheureux et al., 2019). Globally, there are 239,000 new cases and 152,000 deaths every year, making OC the seventh most common cancer and the second most common cause of gynecologic cancer-related mortality (Lheureux et al., 2019). The most common type of OC is epithelial ovarian cancer (EOC). Cytoreduction and combination chemotherapies were performed to treat OC, but the prognosis remains poor (Liu et al., 2019a; Yao et al., 2019; Liu et al., 2020). Recently, tumor biomarkers have been used to monitor the progression and predict the prognosis of EOC, but these biomarkers are not very accurate (Liu et al., 2019a; Yao et al., 2019; Liu et al., 2020).

In our previous study, we found that non-muscle myosin heavy chain $\mathrm{B}$ (MYH10) is an independent prognostic biomarker of EOC (in print). Furthermore, we used the Biogrid website to predict the candidate interacting proteins of MYH10, and we found some candidate proteins that may closely correlate with MYH10, such as MYL9 (Deng et al., 2021), MACF1 (Liu et al., 2021), MYH9 (Liu et al., 2019b), and so on. In the further study, we found that the co-expression of GRK5 (G protein-coupled receptor kinase 5) and ACTC1 (actin alpha cardiac muscle 1) is indeed independent prognostic biomarkers, which indicates their important role in EOC. GRK5 is one of the G protein-coupled receptor kinase (GRK) family members (Gambardella et al., 2016; Jiang et al., 2018; Zhao et al., 2019a; Lagman et al., 2019; Sommer et al., 2019), which is a candidate interacting protein of MYH10. GRK5 can regulate GPCR signaling, which correlates with various diseases like cardiac dysfunction, diabetes, hypertension, Alzheimer's disease, and cancers (Kim et al., 2012; Komolov et al., 2017; Jiang et al., 2018; Alshabi et al., 2019; Zhao et al., 2019b). GRK5 functions as oncogenes in glioblastoma (GBM) (Yang et al., 2018), prostate (Kim et al., 2012), pancreas (Tseng and Zhang, 2000), nonsmall-cell lung (Jiang et al., 2018), and breast (Sommer et al., 2019) cancers. However, to the best of our knowledge, the role of GRK5 in EOC has not been reported.

Similar to GRK5, ACTC1 is also a cardiac-related gene (Kondrashov et al., 2018), and both of them are the candidate interacting proteins of MYH10. ACTC1 encodes cardiac actin, and a mutation at c.G301A causes hypertrophic cardiomyopathy and, in some cases, sudden cardiac death (Frank et al., 2019). Recently, some reports (Kim et al., 2019; da Rocha et al., 2019; Wanibuchi et al., 2018; Cheung et al., 2017; Li et al., 2017; Ohtaki et al., 2017; Zaravinos et al., 2011) have demonstrated that ACTC1 plays an important role in human colon cancer, oral squamous cell carcinoma, GBM, and so on. However, the role of ACTC1 in EOC has not been reported, and the relationship of GRK5 and ACTC1 and EOC has not been explored yet.
The present study identified that GRK5 and ACTC1 were both upregulated in EOC. More importantly, GRK5+/ACTC1+ coexpression was an independent prognostic factor. The GRK5+l ACTC1+ co-expression could predict the development, metastasis, and prognosis of EOC.

\section{MATERIALS AND METHODS}

\section{Paraffin-Embedded Tissue Sections}

Between December 2009 and March 2017, a total of 172 paraffinembedded EOC tissues and 41 matched paraffin-embedded paratumor tissues (the distance away from the margin of the cancer tissue is more than $1.0 \mathrm{~cm}$ ) that had been pathologically confirmed at the memorial hospital of Sun Yat-sen University were collected for the present study. The survival duration was calculated from the date of surgery to November 1, 2018 (last follow-up). The approval of the present study was obtained from the Ethics Committee of the memorial hospital of Sun Yat-sen University. All of the patients provided written informed consent prior to the operation.

\section{Fresh Tissue Specimens}

Between August 2013 and June 2019, 16 fresh EOC tissues and their matched fresh paratumor tissues were collected from the Integrated Hospital of Traditional Chinese Medicine, Southern Medical University, at the time of diagnosis after surgery. All fresh samples were immediately preserved in liquid nitrogen. Approval was obtained from the Ethics Committee of the Integrated Hospital of Traditional Chinese Medicine, Southern Medical University. All of the patients provided written informed consent prior to surgery.

\section{Immunohistochemistry}

The expression of GRK5 and ACTC1 in paraffin-embedded EOC and paired paratumor tissues was detected by IHC staining. First, 4$\mu \mathrm{m}$ paraffin-embedded sections were baked at $65^{\circ} \mathrm{C}$ for $2 \mathrm{~h}$, deparaffinized with xylene, and rehydrated; high tension was used for antigen retrieval, and the specimens were treated with $3 \%$ hydrogen peroxide in methanol, followed by incubation with $1 \%$ bovine serum albumin to block non-specific binding. Subsequently, the samples were incubated with anti-rabbit GRK5 polyclonal (1:150 dilution; Cat. 17032-1-AP; Proteintech) or ACTC1 antibodies (1:200 dilution; Cat. 66125-1-IG; Proteintech) at $4^{\circ} \mathrm{C}$ overnight. Next, the samples were treated with secondary antibody (OriGene, Rockville, MD, USA) and then incubated with streptavidin horseradish peroxidase complex (OriGene, Rockville, MD, USA), immersed in 3-amino-9-ethyl carbazole. The sections were then counterstained with $10 \%$ Mayer's hematoxylin, dehydrated, and mounted in Crystal Mount. Two pathologists evaluated the score of immunostaining for each section. The score was based on the proportion of positively stained cancer cells and the staining intensity. The percentage was scored as follows: samples with $<10 \%$ positive cancer cells were scored as $0 ; 10-50 \%$ were scored as $1,50-75 \%$ were scored as 2 , and $>75 \%$ were scored as 3 . Furthermore, the tissues were classified into four grades based on 

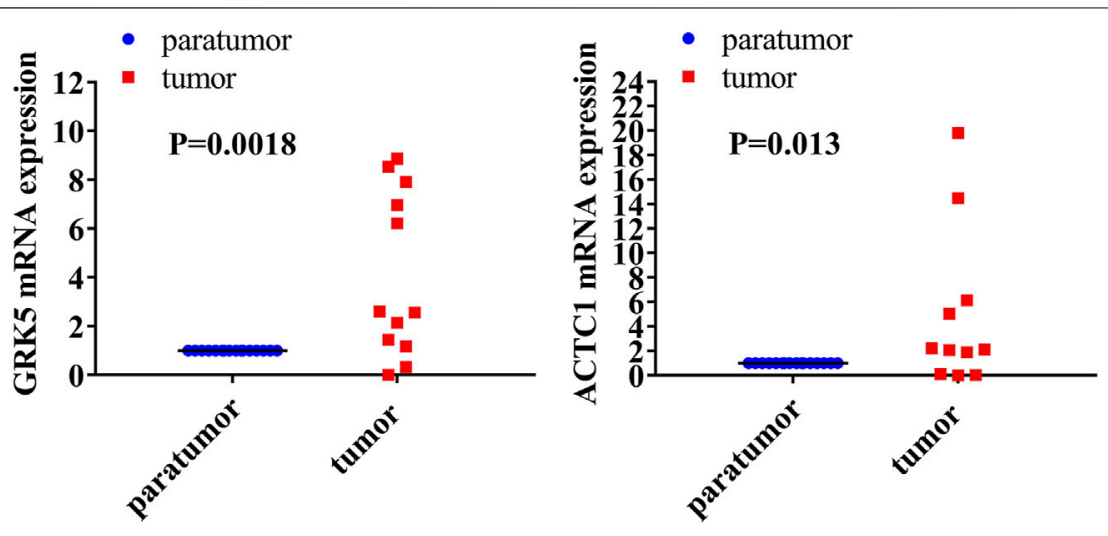

FIGURE 1 | Both GRK5 and ACTC1 were significantly upregulated in EOC tissues compared with that in paratumor tissues using RT-qPCR analysis.

TABLE 1 | Both GRK5 and ACTC1 were significantly upregulated in EOC tissues compared with that in paratumor tissues.

\section{Group}

All of paratumor tissues (total of 16 cases)

Patient 1

Patient 2

Patient 3

Patient 4

Patient 5

Patient 6

Patient 7

Patient 8

Patient 9

Patient 10

Patient 11

Patient 12

Patient 13

Patient 14

Patient 15

Patient 16

Expression of all patients

\section{GRK5 mRNA expression}

$$
\begin{gathered}
1.00 \pm 0.00 \\
6.218 \pm 4.482 \\
6.963 \pm 3.018 \\
8.547 \pm 2.435 \\
8.865 \pm 1.137 \\
2.610 \pm 0.800 \\
2.149 \pm 0.737 \\
1.442 \pm 0.489 \\
29.440 \pm 31.740 \\
0.002 \pm 0.145 \\
96.510 \pm 58.720 \\
2.564 \pm 2.011 \\
0.326 \pm 0.116 \\
1.173 \pm 0.165 \\
7.917 \pm 5.036 \\
22.360 \pm 9.835 \\
148.300 \pm 30.390 \\
21.590 \pm 43.210^{\star \star} \\
\mathbf{0 . 0 0 1 8}
\end{gathered}
$$

\section{ACTC1 mRNA expression}

$$
\begin{aligned}
& 1.00 \pm 0.00 \\
& 14.480 \pm 5.654 \\
& 5.057 \pm 3.370 \\
& 166.100 \pm 105.700 \\
& 833.800 \pm 584.800 \\
& 0.115 \pm 0.075 \\
& 0.016 \pm 0.004 \\
& 2.229 \pm 0.837 \\
& 53.560 \pm 20.970 \\
& 0.029 \pm 0.010 \\
& 246.700 \pm 29.080 \\
& 1.885 \pm 0.405 \\
& 2.079 \pm 0.790 \\
& 6.146 \pm 1.582 \\
& 2.116 \pm 0.705 \\
& 19.800 \pm 2.400 \\
& 2,575.000 \pm 312.400 \\
& 245.600 \pm 656.600^{*} \\
& 0.013
\end{aligned}
$$

ACTC1, actin alpha cardiac muscle 1; GRK5, G protein-coupled receptor kinase 5. Bold value indicates the significant differences.

staining intensity: 0 indicated no staining, 1 indicated weak staining, 2 indicated moderate staining, and 3 indicated strong staining. The staining index (0-9) was calculated as the product of the proportion of positive cells multiplied by the staining intensity score. The best cutoff value was defined as follows: a staining score of $\geq 6$ was considered to indicate high GRK5 or ATCT1 protein expression (also called GRK5+ or ACTC1+), and a staining score of $\leq 5$ indicated low GRK5 or ATCT1 protein expression (also called GRK5- or ACTC1-) (Fu et al., 2017; Zhen et al., 2017; Zhao et al., 2018; Liang et al., 2019; Zou et al., 2019).

\section{Real-Time Quantitative Polymerase Chain Reaction}

The total RNA was extracted from the EOC tissues and paratumor tissues by using TRIzol (Takara Bio, Inc., Shiga, Japan). GAPDH mRNA was detected as the internal control
(Liu et al., 2020). The expression levels of each matched fresh paratumor tissue sample were set as the control group (the expression levels of MYL9 in all of the paratumor tissues were $1.00 \pm 0.00)$, and the relative expression is $2^{-\Delta \Delta C t}$. The thermocycling conditions (Zhao et al., 2016; Liu et al., 2019c; Li et al., 2019; Lin et al., 2019; Xiao et al., 2019) were $95^{\circ} \mathrm{C}$ for $10 \mathrm{~min}$ to activate DNA polymerase, followed by 45 cycles of $95^{\circ} \mathrm{C}$ for $15 \mathrm{~s}, 60^{\circ} \mathrm{C}$ for $15 \mathrm{~s}$, and $72^{\circ} \mathrm{C}$ for $10 \mathrm{~s}$. The specificity of amplification products was confirmed by melting curve analysis. Independent experiments were performed in triplicate. The specific primer sequences were as follows: GRK5 forward, 5'-CCTCCGAAGGACCATAGACA- $3^{\prime}$ and reverse, 5'-GACTGGGGACTTTGGAGTGA-3'; ACTC1 forward, 5'-GGTGATGAAGCCCAGAGCAA-3' and reverse, 5'-GTGGTGACAAAGGAGTAGCC-3'; GAPDH forward, 5' CCATCTTCCAGGAGCGAGAT- $3^{\prime}$ and reverse, $5^{\prime}$-TGCTGA TGATCTTGAGGCTG-3’. 


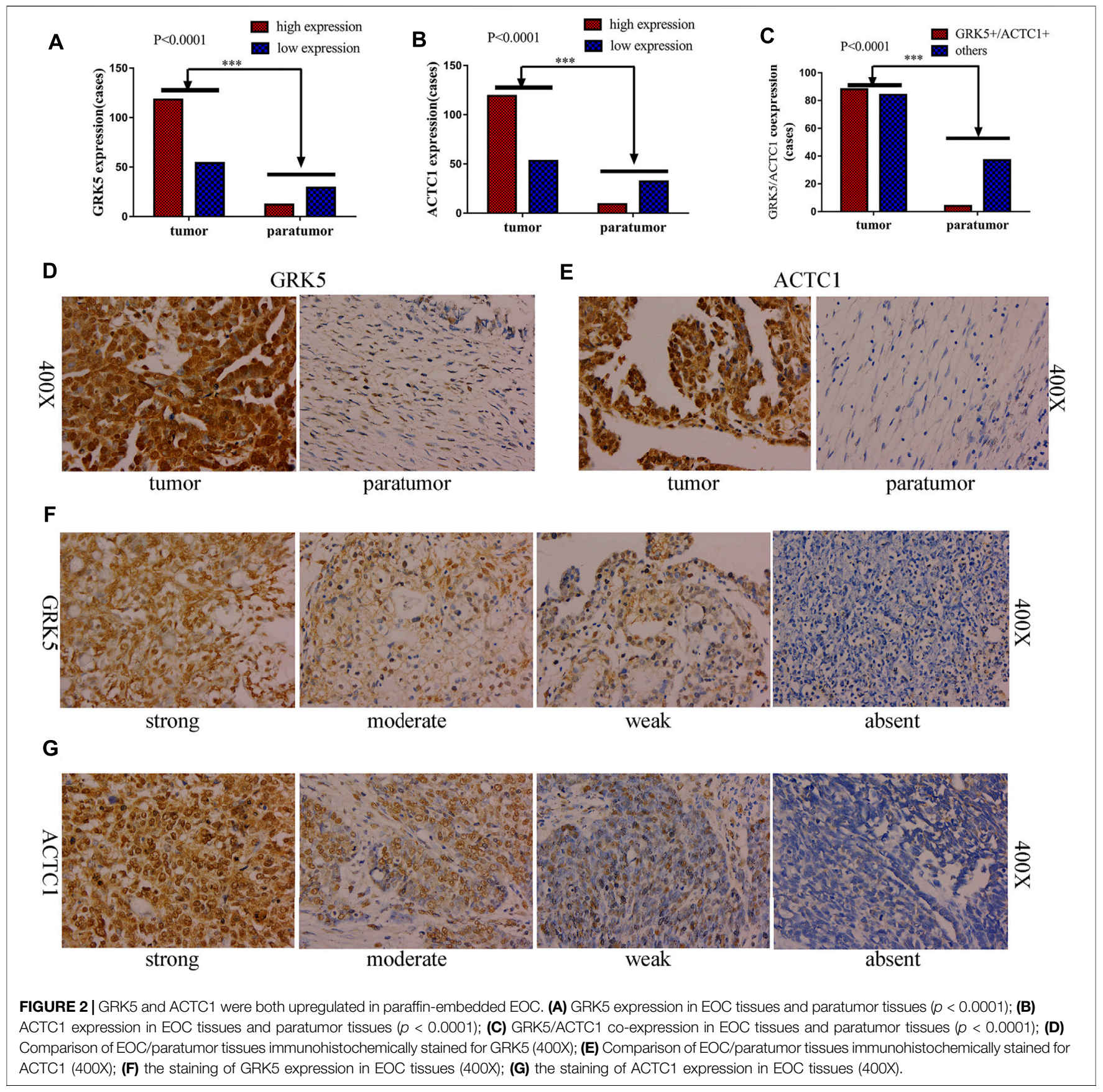

\section{Statistical Analysis}

All data analyses were performed using the statistical software package SPSS 21.0 (IBM Corp.) and GraphPad Prism 7 (GraphPad Software, Inc.). The mRNA expression of GRK5 or ACTC1 was expressed as the mean \pm standard deviation. A two-tailed Student's t-test was used for comparisons between two independent groups (the expression of GRK5 or ACTC1 in paratumor tissues as the control group). The chi-square test or Fisher's exact test was used to analyze the association among GRK5 or ACTC1 or GRK5/ACTC1 co-expression (including GRK5+/ACTC1+, GRK5-/ACTC1+, GRK5+/ ACTC1-, and GRK5-/ACTC1-) and clinicopathological parameters. Furthermore, the recurrence-free survival (RFS) and overall survival (OS) were analyzed by Kaplan-Meier analysis, and the differences were assessed using the log-rank test. Cox's proportional hazards regression model was used for univariate and multivariate analysis. Spearman or Pearson correlation was used for the correlation between GRK5 and ACTC1 expression. $p<0.05$ was considered to indicate statistical significance. 


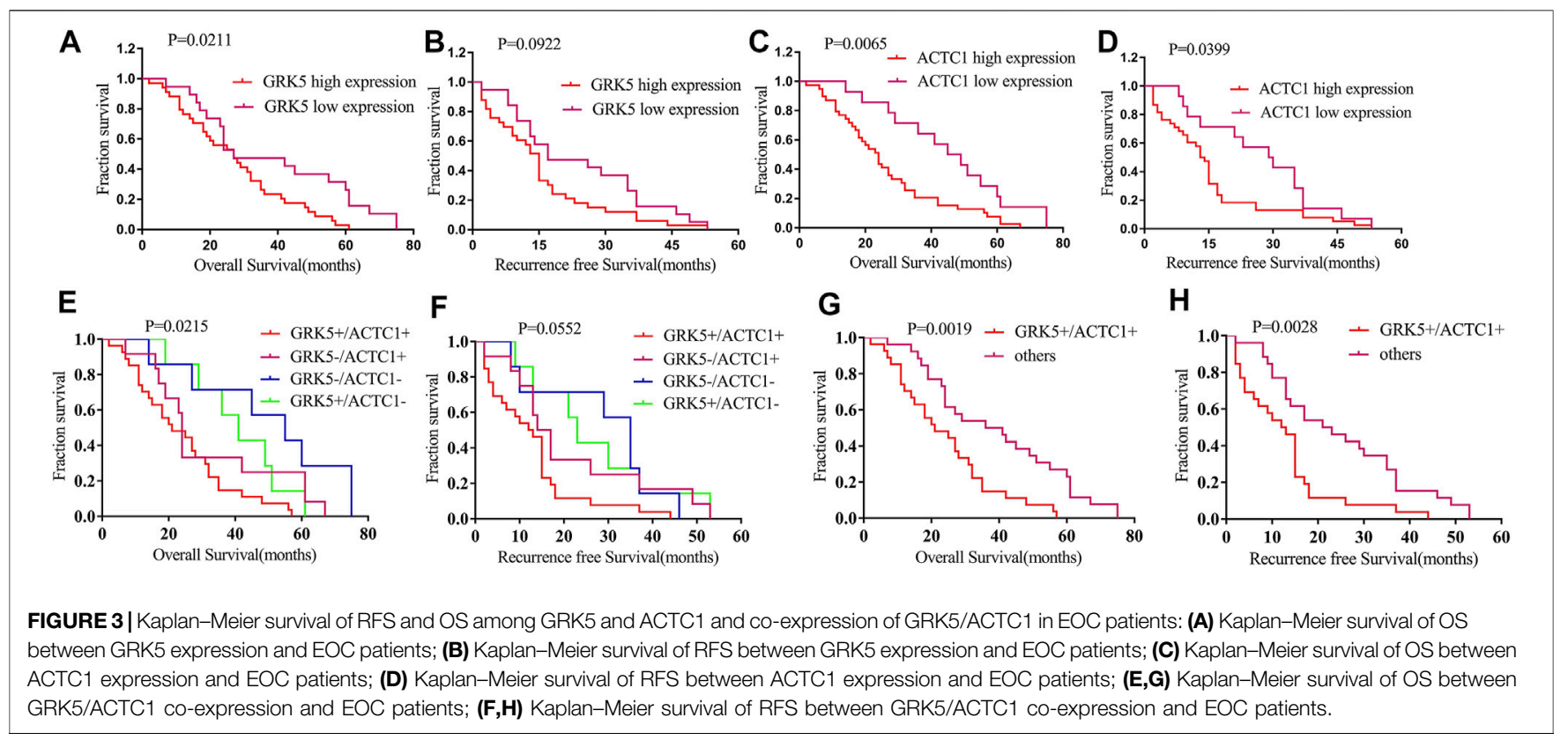

\section{RESULTS}

\section{GRK5 and ACTC1 mRNA Were Both Upregulated in Fresh Epithelial Ovarian Cancer Tissues Compared With That in Paratumor Tissues}

Reverse-transcription quantitative PCR (RT-qPCR) analysis was performed to detect the mRNA expression levels of both GRK5 and ACTC1 in 16 fresh EOC tissues and matched paratumor tissues (Figure 1). The expression of GRK5 or ACTC1 in all of the matched paratumor tissues was set as $1.00 \pm 0.00$, and the expression in each of the tumor tissues was compared with that in the matched paratumor tissues. The results indicated that the mean expression of GRK5 and ACTC1 in the 16 fresh EOC tissues was 21.590 and 245.600, respectively. There was a significant difference between EOC tissues and paratumor tissues $(p=0.0018 ; p=0.013$; Table 1 and Figure 1).

\section{GRK5 and ACTC1 Expression Were Both Assessed in Paraffin-Embedded Epithelial Ovarian Cancer Tissues and Paratumor Tissues by Immunohistochemistry}

To further determine whether GRK5 or ACTC1 protein is upregulated in EOC, 172 paraffin-embedded EOC tissues and 41 matched paratumor tissues were subjected to the IHC analysis of GRK5 and ACTC1 expression. The results indicated that 54/ $172(31.40 \%$, GRK5) and 53/172 (30.81\%, ACTC1) of the cancer samples had low/absent staining (rated as low expression) and $118 / 172$ (68.60\%, GRK5) and $119 / 172$ (69.19\%, ACTC1) had moderate/strong staining (rated as high expression), while the IHC analysis of the 41 paratumor tissues indicated that $29 / 41$ (70.73\%, GRK5) and 32/41 (78.05\%, ACTC1) of the paratumor samples had low/absent staining and 12/41 (29.27\%, GRK5) and 9/41 (21.95\%, ACTC1) had moderate/strong staining. Moreover, the results indicated that $88 / 172(51.16 \%$, GRK5+/ACTC $1+)$ of the cancer samples had moderate/strong staining and 84/172 (48.84\%, others) had low/absent staining, while the IHC analysis of the 41 paratumor tissues indicated that $37 / 41$ (90.24\%, others) and low/absent staining and 4/41 (9.76\%, GRK5+/ACTC1+) had moderate/strong staining. There was a significant difference on the GRK5, ACTC1, and GRK5/ACTC1 expression between the cancer and paratumor tissues $(p<0.0001, p<0.0001, p<0.0001$; Figures 2A-C). Furthermore, it was observed that GRK5 and ACTC1 proteins were both located in the nucleus (Figures 2D-G).

\section{GRK5 or ACTC1 or GRK5/ \\ ACTC1 Co-expression Was Associated With Recurrence-Free Survival and Overall Survival of Epithelial Ovarian Cancer Patients}

In this study, patients with GRK5+ exhibited a median OS time of 27 months, while patients with GRK5- exhibited a median OS time of 27 months $(\mathrm{HR}=1.81,95 \% \mathrm{CI}: 1.056-3.101)$. Patients with GRK5+ exhibited a median RFS time of only 15 months, while patients with GRK5- exhibited a median RFS time of 17 months $(\mathrm{HR}=1.555,95 \% \mathrm{CI}:$ 0.9023-2.681). Moreover, patients with ACTC1+ exhibited a median OS time of only 24 months, while patients with ACTC1- exhibited a median OS time of 47 months $(\mathrm{HR}=2.159,95 \% \mathrm{CI}: 1.255-3.716)$. Patients with ACTC1+ exhibited a median RFS time of only 14 months, while patients with ACTC1- exhibited a median RFS time of 29.5 months (HR = 1.784, 95\% CI: 1.024-3.11). In addition, patients with GRK5+/ ACTC1+ co-expression exhibited a median OS time of only 21.0 months, while patients with others co-expression exhibited a mean 
TABLE 2 | GRK5, ACTC1 and GRK5/ACTC1 co-expression in association with clinical parameters of EOC.

\begin{tabular}{|c|c|c|c|c|c|c|c|c|c|c|c|}
\hline \multirow[t]{2}{*}{ Parameters } & & \multirow[t]{2}{*}{ Total } & \multicolumn{3}{|c|}{ GRK5 } & \multicolumn{3}{|c|}{ ACTC1 } & \multicolumn{3}{|c|}{ Co-expression of GRK5/ACTC1 } \\
\hline & & & Low & High & $\begin{array}{c}p \text {-value ( } \chi^{2} \text { or } \\
\text { Fisher's exact } \\
\text { test) }\end{array}$ & Low & High & $\begin{array}{c}p \text {-value ( } \chi^{2} \text { or } \\
\text { Fisher's exact } \\
\text { test) }\end{array}$ & $\begin{array}{l}\text { GRK5+/ } \\
\text { ACTC1+ }\end{array}$ & Others & $\begin{array}{c}p \text {-value ( } \chi 2 \text { or } \\
\text { Fisher's exact } \\
\text { test) }\end{array}$ \\
\hline \multirow[t]{2}{*}{ Age (years) } & $\leq 50$ & 75 & 27 & 48 & 0.2525 & 25 & 50 & 0.5292 & 33 & 42 & 0.0984 \\
\hline & $>50$ & 97 & 27 & 70 & & 28 & 69 & & 55 & 42 & \\
\hline \multirow[t]{4}{*}{ Histology } & Serous & 110 & 29 & 81 & 0.0580 & 29 & 81 & 0.0729 & 65 & 45 & 0.0095 \\
\hline & Mucoid & 10 & 6 & 4 & & 5 & 5 & & 2 & 8 & \\
\hline & Endometrial & 22 & 10 & 12 & & 7 & 15 & & 7 & 15 & \\
\hline & Clear cell & 12 & 5 & 7 & & 7 & 5 & & 4 & 8 & \\
\hline \multirow[t]{2}{*}{ FIGO stage } & I/II & 48 & 24 & 24 & 0.0011 & 25 & 23 & 0.0002 & 11 & 37 & $<0.0001$ \\
\hline & III/IV & 124 & 30 & 94 & & 28 & 96 & & 77 & 47 & \\
\hline \multirow{2}{*}{$\begin{array}{l}\text { Lymph node } \\
\text { metastasis }\end{array}$} & No & 47 & 23 & 24 & 0.0832 & 24 & 23 & 0.0266 & 12 & 35 & 0.0062 \\
\hline & Yes & 28 & 8 & 20 & & 7 & 21 & & 16 & 12 & \\
\hline \multirow{2}{*}{$\begin{array}{l}\text { Intraperitoneal } \\
\text { metastasis }\end{array}$} & No & 57 & 25 & 32 & 0.0131 & 29 & 28 & $<0.0001$ & 16 & 41 & $<0.0001$ \\
\hline & Yes & 115 & 29 & 86 & & 24 & 91 & & 72 & 43 & \\
\hline \multirow{2}{*}{$\begin{array}{l}\text { Intestinal } \\
\text { metastasis }\end{array}$} & No & 93 & 44 & 49 & $<0.0001$ & 39 & 54 & 0.0006 & 29 & 64 & $<0.0001$ \\
\hline & Yes & 79 & 10 & 69 & & 14 & 65 & & 59 & 20 & \\
\hline \multirow[t]{2}{*}{ Vital status } & Alive & 60 & 24 & 36 & 0.6502 & 28 & 32 & 0.0262 & 19 & 41 & 0.0374 \\
\hline & Dead & 53 & 19 & 34 & & 14 & 39 & & 27 & 26 & \\
\hline \multirow{2}{*}{$\begin{array}{l}\text { Intraperitoneal } \\
\text { recurrence }\end{array}$} & No & 122 & 38 & 84 & 0.8558 & 41 & 81 & 0.3497 & 60 & 62 & 0.5503 \\
\hline & Yes & 46 & 15 & 31 & & 12 & 34 & & 25 & 21 & \\
\hline \multirow[t]{2}{*}{ Distant recurrence } & No & 140 & 45 & 95 & 0.7105 & 47 & 93 & 0.2069 & 69 & 71 & 0.4478 \\
\hline & Yes & 28 & 8 & 20 & & 6 & 22 & & 16 & 12 & \\
\hline \multirow{2}{*}{$\begin{array}{l}\text { Differentiation } \\
\text { grade }\end{array}$} & G1/G2 & 58 & 26 & 32 & 0.0029 & 21 & 37 & 0.1418 & 22 & 36 & 0.0046 \\
\hline & G3 & 103 & 23 & 80 & & 26 & 77 & & 63 & 40 & \\
\hline \multirow{2}{*}{$\begin{array}{l}\text { Platinum } \\
\text { resistance }\end{array}$} & No & 164 & 50 & 114 & 0.1784 & 50 & 114 & 0.6438 & 84 & 80 & 0.6782 \\
\hline & Yes & 5 & 3 & 2 & & 2 & 3 & & 2 & 3 & \\
\hline \multirow{2}{*}{$\begin{array}{l}\text { Ascites with tumor } \\
\text { cells }\end{array}$} & No & 45 & 22 & 23 & 0.0077 & 16 & 29 & 0.5082 & 16 & 29 & 0.0153 \\
\hline & Yes & 35 & 7 & 28 & & 10 & 25 & & 22 & 13 & \\
\hline \multirow[t]{2}{*}{ CA125 (U/ml) } & $\leq 35$ & 22 & 7 & 15 & 0.8794 & 4 & 18 & 0.3150 & 13 & 9 & 0.4464 \\
\hline & $>35$ & 139 & 42 & 97 & & 44 & 95 & & 70 & 69 & \\
\hline \multirow[t]{2}{*}{ CA72-4 (U/ml) } & $\leq 7$ & 69 & 23 & 46 & 0.5078 & 22 & 47 & 0.6315 & 33 & 36 & 0.3135 \\
\hline & $>7$ & 71 & 20 & 51 & & 20 & 51 & & 40 & 31 & \\
\hline \multirow[t]{2}{*}{ CA153 (U/ml) } & $\leq 25$ & 11 & 5 & 6 & 0.3095 & 3 & 8 & $>0.9999$ & 6 & 5 & 0.9267 \\
\hline & $>25$ & 41 & 12 & 29 & & 10 & 31 & & 23 & 18 & \\
\hline \multirow[t]{2}{*}{ AFP (U/ml) } & $\leq 25$ & 130 & 40 & 90 & $>0.9999$ & 40 & 90 & $>0.9999$ & 66 & 64 & $>0.9999$ \\
\hline & $>25$ & 1 & 0 & 1 & & 0 & 1 & & 1 & 0 & \\
\hline \multirow[t]{2}{*}{ CEA (U/ml) } & $\leq 5$ & 117 & 35 & 82 & 0.8534 & 34 & 83 & 0.1893 & 61 & 56 & 0.5433 \\
\hline & $>5$ & 18 & 5 & 13 & & 8 & 10 & & 8 & 10 & \\
\hline \multirow[t]{2}{*}{ HE4 (U/ml) } & $\leq 140$ & 30 & 8 & 22 & 0.6835 & 9 & 21 & 0.5101 & 17 & 13 & 0.2746 \\
\hline & $>140$ & 65 & 20 & 45 & & 24 & 41 & & 29 & 36 & \\
\hline
\end{tabular}

ACTC1, actin alpha cardiac muscle 1; FIGO, International Federation of Gynecology and Obstetrics; GRK5, G protein-coupled receptor kinase 5. Bold values indicate the significant differences.

OS time of 38.5 months (HR $=2.17,95 \%$ CI: $1.22-3.858$ ). Patients with GRK5+/ACTC1+ co-expression exhibited a median RFS time of only 12.5 months, while patients with other coexpressions exhibited a mean RFS time of 22 months ( $\mathrm{HR}=$ 2.092, 95\% CI: 1.17-3.739). Kaplan-Meier survival analysis demonstrated that there was a statistical significance on the OS and RFS between GRK5+/ACTC1+ and others coexpression $(p=0.0019$ and $p=0.0028$, respectively), and there was also a statistical significance on the OS and RFS between ACTC1+ and ACTC1- $(p=0.0065$ and $p=0.0399$, respectively). However, there was a significant difference between the GRK5+ and GRK5- on OS $(p=0.0211)$, but there was no significance on the RFS between GRK5+ and GRK5- $(p=0.0922)$ (Figure 3). A survival analysis showed that the cumulative OS and RFS rates of EOC patients increased with the increase in GRK5+/ACTC1+ co-expression (Figure 3). 
TABLE 3 | Correlation between GRK5 and ACTC1 expression.

\begin{tabular}{lcccccc}
\hline GRK5 & \multicolumn{2}{c}{ ACTC1 } & & Spearman's R & \multirow{2}{*}{$\mathbf{2}$} & $\boldsymbol{p}$ \\
\cline { 2 - 3 } & High & Low & & & & \\
\hline high & 88 & 30 & 0.173 & 5.122 & 0.0236 \\
Low & 31 & 23 & & & \\
\hline
\end{tabular}

ACTC1, actin alpha cardiac muscle 1; GRK5, G protein-coupled receptor kinase 5

\section{GRK5 and ACTC1 and GRK5/ \\ ACTC1 Co-expression Were Associated With the Clinicopathological Parameters of Epithelial Ovarian Cancer Patients}

Subsequently, we evaluated their correlation with the clinicopathological parameters of EOC patients. The results of using $\chi 2$ or Fisher's exact test showed that there were significant relationships between GRK5 expression and clinicopathological parameters of EOC, such as the following factors: FIGO (International Federation of Gynecology and Obstetrics) stage, intraperitoneal metastasis, intestinal metastasis, differentiation grade, ascites with tumor cells, and so on (Table 2). There were significant relationships between ACTC1 expression and the clinicopathological parameters of EOC, such as the following factors: FIGO stage, lymph node metastasis, intraperitoneal metastasis, intestinal metastasis, vital status, and so on (Table 2). At last, we used $\chi 2$ or Fisher's exact test to explore the relationship between GRK5/ACTC1 coexpression and the clinicopathological parameters of EOC, and we found that there were significant differences in the following factors: histology, FIGO stage, lymph node metastasis, intraperitoneal metastasis, intestinal metastasis, vital status, differentiation grade, ascites with tumor cells, and so on (Table 2).

\section{Correlation Between GRK5 and ACTC1 Expression}

To explore the relationship between GRK5 and ACTC1, Spearman correlation and $\chi 2$ tests were used for analysis, and the results showed that there is a statistical significance between them $(p=0.0236)$ (Table 3).

\section{GRK5 mRNA Was Positively Correlated With ACTC1 mRNA}

Further, we assessed the correlation between GRK5 and ACTC1 mRNA expression. Using Pearson correlation analysis, we found that GRK5 was positively correlated with ACTC1 $(r=0.8174, p=$ 0.0001) (Figure 4).

\section{Co-Expression of GRK5+/ACTC1+ Was a Useful Independent Prognostic Predictor of Epithelial Ovarian Cancer}

Furthermore, we also assessed the prognostic value of GRK5, ACTC1, and GRK5/ACTC1 co-expression in EOC patients. In

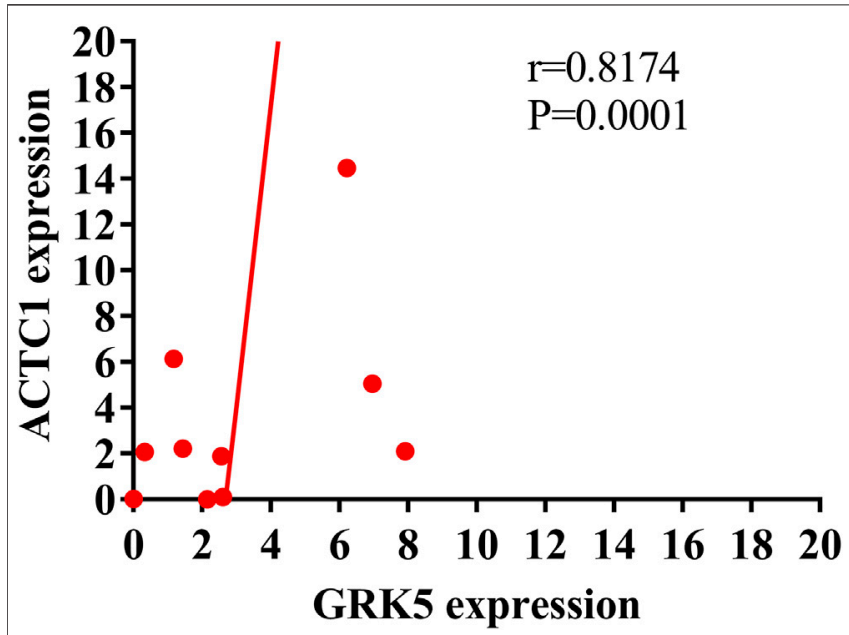

FIGURE 4 | Pearson correlation analysis showing the positive correlation of GRK5 mRNA and ACTC1 mRNA.

a univariate Cox model analysis, GRK5, ACTC1, and GRK5/ ACTC1 co-expression; intestinal metastasis; postoperative chemotherapy; platinum resistance; and hyperthermic intraperitoneal chemotherapy (HIPEC) were significant prognostic factors (Table 4). Moreover, in a multivariate Cox regression model, we found that GRK5+/ACTC1+ coexpression, intestinal metastasis, postoperative chemotherapy, platinum resistance, and HIPEC were indeed independent prognostic factors of EOC (Table 4), but GRK5 expression and ACTC1 expression were no longer significant.

\section{DISCUSSIONS}

In our previous study, we found that MYH10 is an independent prognostic biomarker of EOC (in print). Furthermore, we used the Biogrid website to predict the candidate interacting proteins of MYH10, and we found some candidate proteins that may closely correlate with MYH10, such as MYL9 (Deng et al., 2021), MACF1 (Liu et al., 2021), MYH9 (Liu et al., 2019b), and so on. In the further study, we found that the co-expression of GRK5 and ACTC1 is indeed independent prognostic biomarkers, which indicates their important role in EOC. GRK5 plays oncogenic roles in GBM, prostate, pancreas, renal cell, nonsmall-cell lung, and breast cancers (Kim et al., 2012; Gambardella et al., 2016; Komolov et al., 2017; Jiang et al., 2018; Yang et al., 2018; Alshabi et al., 2019; Zhao et al., 2019a; Zhao et al., 2019b; Lagman et al., 2019; Sommer et al., 2019). It is clear (Gambardella et al., 2016) that when GRK5 is localized at the plasma membrane, it often exerts an anti-tumoral effect, attenuating growth-associated signaling pathways through its ability to desensitize GPCR and non-GPCR receptors. However, when GRK5 moves to cytosol or nucleus, it often promotes tumor growth acting on nonreceptor substrates. Consistent with previous findings (Kim et al., 2012; Jiang et al., 2018; Alshabi 
TABLE 4 | Cox regression univariate and multivariate analyses of prognostic factors in EOC.

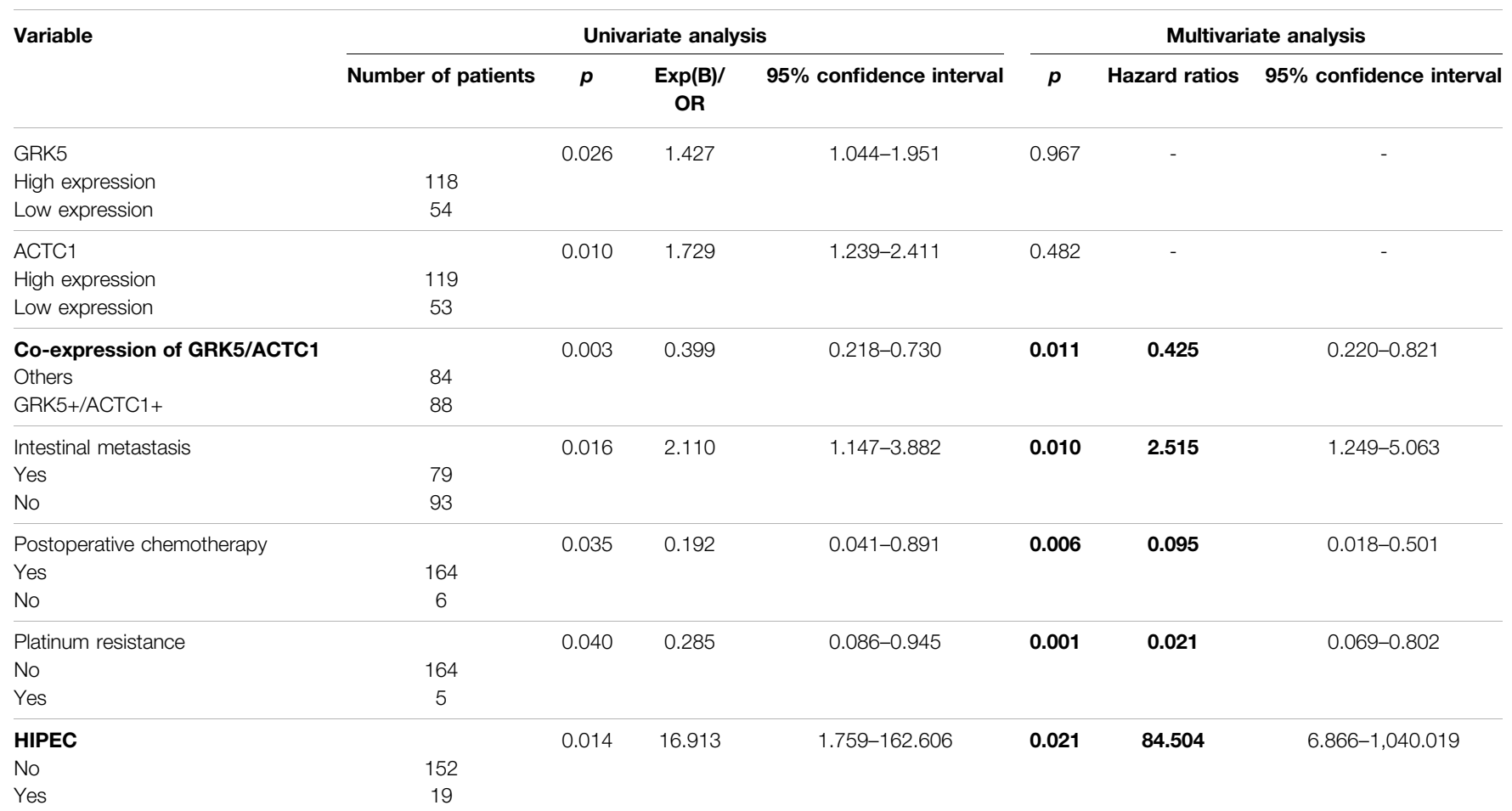

ACTC1, actin alpha cardiac muscle 1; GRK5, G protein-coupled receptor kinase 5; HIPEC, hyperthermic intraperitoneal chemotherapy. Bold values indicate the significant differences.

et al., 2019; Zhao et al., 2019a), in the present study, our results showed that GRK5 was upregulated in paraffin-embedded and fresh EOC tissues, and it is located mainly at the nucleus, which suggested that GRK5 may play a candidate oncogenic role in EOC. Further, Kaplan-Meier survival analysis showed that GRK5 high expression was associated with shorter OS, but not RFS, which is consistent with the previous reports (Jiang et al., 2018; Zhao et al., 2019a) that GRK5 high-expression NSCLC or renal cell carcinoma patients had a worse OS rate than the low-expression patients. Furthermore, GRK5 expression was associated with the following clinicopathological parameters, such as: FIGO stage, intraperitoneal metastasis, intestinal metastasis, differentiation grade, and ascites with tumor cells, which showed that GRK5 high expression was closely related with the development and metastasis of EOC.

Similar to GRK5, ACTC1 is also a cardiac-related gene (Kondrashov et al., 2018), and both of them are the candidate interacting proteins of MYH10. ACTC1 encodes the cardiac form of actin (Kondrashov et al., 2018). Ohtaki et al. reported (Ohtaki et al., 2017) that ACTC1 served as a clinical marker to detect migration and poor prognosis in GBM patients. In addition, our results also demonstrated that ACTC1 was upregulated in paraffin-embedded and fresh EOC tissues compared with that in paratumor tissues, which suggested that ACTC1 played a candidate oncogenic role in EOC. This is consistent with the role of ACTC1 (Kim et al., 2019; da Rocha et al., 2019; Wanibuchi et al., 2018; Cheung et al., 2017; Li et al., 2017; Ohtaki et al., 2017; Zaravinos et al., 2011) in GBM, colon, prostate, oral squamous cell and breast cancers. Further, Kaplan-Meier survival analysis showed that ACTC1 high expression was closely associated with shorter OS and RFS, which is consistent with the previous study of ACTC1 in GBM. Furthermore, ACTC1 expression was associated with clinicopathological parameters of EOC, such as: FIGO stage, lymph node metastasis, intraperitoneal metastasis, intestinal metastasis, and vital status, which showed that ACTC1 was also closely associated with the development and metastasis of EOC.

Importantly, ACTC1 and GRK5 are both cardiac-related genes. In this study, our results showed that ACTC1 mRNA and protein expression were both positively correlated with GRK5 mRNA and protein expression, and Kaplan-Meier survival analysis showed that GRK5+/ACTC1+ was closely associated with poor OS and RFS. Multivariate Cox regression analysis showed that GRK5+/ACTC1+ co-expression was an independent prognostic factor rather than GRK5 or ACTC1 alone. Moreover, GRK5-/ACTC1+ patients had a worse survival than GRK5+/ACTC1- patients, which suggested that ACTC1 was more closely correlated with survival than GRK5. In addition, GRK5/ACTC1 co-expression was associated with the following factors: histology, FIGO stage, lymph node metastasis, intraperitoneal metastasis, intestinal metastasis, vital status, differentiation grade, and ascites with tumor cells. All of these results suggested that the combination 
of GRK5 and ACTC1 could predict the development, progression, metastasis, and prognosis of EOC more precisely. In future, we need more in vivo (such as the subcutaneous xenograft tumor studies and the lung xenograft tumor studies, and so on) and in vitro research to demonstrate its role and molecular mechanism in development, progression, metastasis, and prognosis of EOC.

\section{CONCLUSION}

Taken together, the results of the present study suggest that GRK5 and ACTC1 are both upregulated in EOC, and GRK5+/ACTC1+ co-expression could predict the development, metastasis, and prognosis of EOC. The co-expression of GRK5+/ACTC1+ can be recommended as prognostic-predicting biomarkers in EOC, and it may provide an important value in the clinical therapy and supervision of EOC.

\section{DATA AVAILABILITY STATEMENT}

The original contributions presented in the study are included in the article/Supplementary Material, further inquiries can be directed to the corresponding author.

\section{REFERENCES}

Alshabi, A. M., Vastrad, B., Shaikh, I. A., and Vastrad, C. (2019). Identification of Important Invasion and Proliferation Related Genes in Adrenocortical Carcinoma. Med. Oncol. 36, 73. doi:10.1007/s12032-019-1296-7

Chen, W., Zheng, R., Baade, P. D., Zhang, S., Zeng, H., Bray, F., et al. (2016). Cancer Statistics in China, 2015. CA: A Cancer J. Clinicians 66, 115-132. doi:10.3322/ caac. 21338

Cheung, A. S., de Rooy, C., Levinger, I., Rana, K., Clarke, M. V., How, J. M., et al. (2017). Actin Alpha Cardiac Muscle 1 Gene Expression Is Upregulated in the Skeletal Muscle of Men Undergoing Androgen Deprivation Therapy for Prostate Cancer. J. Steroid Biochem. Mol. Biol. 174, 56-64. doi:10.1016/ j.jsbmb.2017.07.029

da Rocha, R. G., Santos, E. M. S., Santos, E. M., Gomes, E. S. B., Ramos, G. V., Aguiar, K. M., et al. (2019). Leptin Impairs the Therapeutic Effect of Ionizing Radiation in Oral Squamous Cell Carcinoma Cells. J. Oral Pathol. Med. 48, 17-23. doi:10.1111/jop.12786

Deng, Y., Liu, L., Feng, W., Lin, Z., Ning, Y., and Luo, X. (2021). High Expression of MYL9 Indicates Poor Clinical Prognosis of Epithelial Ovarian Cancer. Pra 16, 533-539. doi:10.2174/1574891X16666210706153740

Frank, D., Yusuf Rangrez, A., Friedrich, C., Dittmann, S., Stallmeyer, B., Yadav, P., et al. (2019). Cardiac a-Actin (ACTC1) Gene Mutation Causes Atrial-Septal Defects Associated with Late-Onset Dilated Cardiomyopathy. Circ. Genom Precis Med. 12, e002491. doi:10.1161/CIRCGEN.119.002491

Fu, Q., Song, X., Liu, Z., Deng, X., Luo, R., Ge, C., et al. (2017). miRomics and Proteomics Reveal a miR-296-3p/PRKCA/FAK/Ras/c-Myc Feedback Loop Modulated by HDGF/DDX5/ $\beta$-catenin Complex in Lung Adenocarcinoma. Clin. Cancer Res. 23, 6336-6350. doi:10.1158/10780432.ccr-16-2813

Gambardella, J., Franco, A., Giudice, C. D., Fiordelisi, A., Cipolletta, E., Ciccarelli, M., et al. (2016). Dual Role of GRK5 in Cancer Development and Progression. Transl Med. Unisa 14, 28-37.

Jiang, L.-P., Fan, S.-Q., Xiong, Q.-X., Zhou, Y.-C., Yang, Z.-Z., Li, G.-F., et al. (2018). GRK5 Functions as an Oncogenic Factor in Non-small-cell Lung Cancer. Cell Death Dis 9, 295. doi:10.1038/s41419-018-0299-1

\section{ETHICS STATEMENT}

The studies involving human participants were reviewed and approved by the Ethics Committee of the Integrated Hospital of Traditional Chinese Medicine, Southern Medical University. The patients/participants provided their written informed consent to participate in this study.

\section{AUTHOR CONTRIBUTIONS}

Conception: JL, PL and CC Interpretation or analysis of data: LL and JL Preparation of the manuscript: LL and JL Revision for important intellectual content: YN and ZL Supervision: CC.

\section{FUNDING}

This work was supported by the Guangdong Basic and Applied Basic Research Foundation (grant no. 2020A1515110030), the Natural Science Foundation of Guangdong Province (grant no. 2020A1515010284), the President funds of Integrated Hospital of Traditional Chinese Medicine, Southern Medical University (No. 1201902001; No. 1201901002), Guangzhou science and Technology Program (grant no. 202102080060).

Kim, E.-K., Song, M.-J., Jung, Y., Lee, W.-S., and Jang, H. H. (2019). Proteomic Analysis of Primary Colon Cancer and Synchronous Solitary Liver Metastasis. Cancer Genomics Proteomics 16, 583-592. doi:10.21873/cgp.20161

Kim, J. I., Chakraborty, P., Wang, Z., and Daaka, Y. (2012). G-protein Coupled Receptor Kinase 5 Regulates Prostate Tumor Growth. J. Urol. 187, 322-329. doi:10.1016/j.juro.2011.09.049

Komolov, K. E., Du, Y., Duc, N. M., Betz, R. M., Rodrigues, J. P. G. L. M., Leib, R. D., et al. (2017). Structural and Functional Analysis of a $\beta 2$-Adrenergic Receptor Complex with GRK5. Cell 169, 407-421. doi:10.1016/j.cell.2017.03.047

Kondrashov, A., Duc Hoang, M., Smith, J. G. W., Bhagwan, J. R., Duncan, G., Mosqueira, D., et al. (2018). Simplified Footprint-free Cas9/CRISPR Editing of Cardiac-Associated Genes in Human Pluripotent Stem Cells. Stem Cell Dev. 27, 391-404. doi:10.1089/scd.2017.0268

Lagman, J., Sayegh, P., Lee, C. S., Sulon, S. M., Jacinto, A. Z., Sok, V., et al. (2019). G Protein-Coupled Receptor Kinase 5 Modifies Cancer Cell Resistance to Paclitaxel. Mol. Cell Biochem 461, 103-118. doi:10.1007/s11010-019-03594-9

Lheureux, S., Braunstein, M., and Oza, A. M. (2019). Epithelial Ovarian Cancer: Evolution of Management in the Era of Precision Medicine. CA. Cancer J. Clin. 69, 280-304. doi:10.3322/caac.21559

Li, Y., Liu, X., Lin, X., Zhao, M., Xiao, Y., Liu, C., et al. (2019). Chemical Compound Cinobufotalin Potently Induces FOXO1-Stimulated Cisplatin Sensitivity by Antagonizing its Binding Partner MYH9. Sig Transduct Target. Ther. 4, 48. doi:10.1038/s41392-019-0084-3

Li, Y., Rong, G., and Kang, H. (2017). Taxotere-induced Elevated Expression of IL8 in Carcinoma-Associated Fibroblasts of Breast Invasive Ductal Cancer. Oncol. Lett. 13, 1856-1860. doi:10.3892/ol.2017.5612

Liang, Z., Liu, Z., Cheng, C., Wang, H., Deng, X., Liu, J., et al. (2019). VPS33B Interacts with NESG1 to Modulate EGFR/PI3K/AKT/c-Myc/P53/miR-133a-3p Signaling and Induce 5-fluorouracil Sensitivity in Nasopharyngeal Carcinoma. Cell Death Dis 10, 305. doi:10.1038/s41419-019-1457-9

Lin, X., Zuo, S., Luo, R., Li, Y., Yu, G., Zou, Y., et al. (2019). HBX-induced miR-5188 Impairs FOXO1 to Stimulate $\beta$-catenin Nuclear Translocation and Promotes Tumor Stemness in Hepatocellular Carcinoma. Theranostics 9, 7583-7598. doi:10.7150/thno.37717

Liu, L., Yi, J., Deng, X., Yuan, J., Zhou, B., Lin, Z., et al. (2019). MYH9 Overexpression Correlates with Clinicopathological Parameters and Poor 
Prognosis of Epithelial Ovarian Cancer. Oncol. Lett. 18 (2), 1049-1056. doi:10.3892/ol.2019.10406

Liu, L., Hu, K., Zeng, Z., Xu, C., Lv, J., Lin, Z., et al. (2021). Expression and Clinical Significance of Microtubule-Actin Cross-Linking Factor 1 in Serous Ovarian Cancer. Pra 16 (1), 66-72. doi:10.2174/1574892816666210211091543

Liu, L., Ning, Y., Yi, J., Yuan, J., Fang, W., Lin, Z., et al. (2020). miR-6089/MYH9/ $\beta$ catenin/c-Jun Negative Feedback Loop Inhibits Ovarian Cancer Carcinogenesis and Progression. Biomed. Pharmacother. 125, 109865. doi:10.1016/ j.biopha.2020.109865

Liu, L., Zeng, Z., Yi, J., Zuo, L., Lv, J., Yuan, J., et al. (2019). Expression and Clinical Significance of Transcription Factor 4 (TCF4) in Epithelial Ovarian Cancer. Cbm 24, 213-221. doi:10.3233/cbm-181849

Liu, Y., Jiang, Q., Liu, X., Lin, X., Tang, Z., Liu, C., et al. (2019). Cinobufotalin Powerfully Reversed EBV-miR-BART22-Induced Cisplatin Resistance via Stimulating MAP2K4 to Antagonize Non-muscle Myosin Heavy Chain IIA/ glycogen Synthase 3 $\beta / \beta$-Catenin Signaling Pathway. EBioMedicine 48 (19), 386-404. doi:10.1016/j.ebiom.2019.08.040

Ohtaki, S., Wanibuchi, M., Kataoka-Sasaki, Y., Sasaki, M., Oka, S., Noshiro, S., et al. (2017). ACTC1 as an Invasion and Prognosis Marker in Glioma. Jns 126, 467-475. doi:10.3171/2016.1.jns152075

Sommer, A.-K., Falcenberg, M., Ljepoja, B., Fröhlich, T., Arnold, G. J., Wagner, E., et al. (2019). Downregulation of GRK5 Hampers the Migration of Breast Cancer Cells. Sci. Rep. 9, 15548. doi:10.1038/s41598-019-51923-1

Tseng, C.-C., and Zhang, X.-Y. (2000). Role of G Protein-Coupled Receptor Kinases in Glucose-dependent Insulinotropic Polypeptide Receptor Signaling*. Endocrinology 141, 947-952. doi:10.1210/endo.141.3.7365

Wanibuchi, M., Ohtaki, S., Ookawa, S., Kataoka-Sasaki, Y., Sasaki, M., Oka, S., et al. (2018). Actin, Alpha, Cardiac Muscle 1 (ACTC1) Knockdown Inhibits the Migration of Glioblastoma Cells In Vitro. J. Neurol. Sci. 392, 117-121. doi:10.1016/j.jns.2018.07.013

Xiao, Y. Y., Lin, L., Li, Y. H., Jiang, H. P., Zhu, L. T., Deng, Y. R., et al. (2019). ZEB1 Promotes Invasion and Metastasis of Endometrial Cancer by Interacting with HDGF and Inducing its Transcription. Am. J. Cancer Res. 9, 2314-2330.

Yang, Y., Wu, J. J., Cheng, C. D., Bao, D. J., Dong, Y. F., Li, D. X., et al. (2018). G-protein-coupled Receptor Kinase-5 Promotes Glioblastoma Progression by Targeting the Nuclear Factor Kappa B Pathway. Am. J. Transl Res. 10, 3370-3384.

Yao, Y., Liu, L., He, W., Lin, X., Zhang, X., Lin, Z., et al. (2019). Low Expression of KIF7 Indicates Poor Prognosis in Epithelial Ovarian Cancer. Cbm 26, 481-489. doi:10.3233/CBM-190328

Zaravinos, A., Lambrou, G. I., Boulalas, I., Delakas, D., and Spandidos, D. A. (2011). Identification of Common Differentially Expressed Genes in Urinary Bladder Cancer. PLoS One 6, e18135. doi:10.1371/journal.pone.0018135
Zhao, J., Li, X., Chen, X., Cai, Y., Wang, Y., Sun, W., et al. (2019). GRK5 Influences the Phosphorylation of Tau via GSK3 $\beta$ and Contributes to Alzheimer's Disease. J. Cell Physiol 234, 10411-10420. doi:10.1002/jcp.27709

Zhao, M., Luo, R., Liu, Y., Gao, L., Fu, Z., Fu, Q., et al. (2016). miR-3188 Regulates Nasopharyngeal Carcinoma Proliferation and Chemosensitivity through a FOXO1-Modulated Positive Feedback Loop with mTOR-P-Pi3k/akt-C-JUN. Nat. Commun. 7, 11309. doi:10.1038/ncomms11309

Zhao, M., Xu, P., Liu, Z., Zhen, Y., Chen, Y., Liu, Y., et al. (2018). RETRACTED ARTICLE: Dual Roles of miR-374a by Modulated C-Jun Respectively Targets CCND1-Inducing PI3K/AKT Signal and PTEN-Suppressing Wnt/ $\beta$-Catenin Signaling in Non-small-cell Lung Cancer. Cell Death Dis 9, 78. doi:10.1038/ s41419-017-0103-7

Zhao, T. L., Gan, X. X., Bao, Y., Wang, W. P., Liu, B., and Wang, L. H. (2019). GRK5 Promotes Tumor Progression in Renal Cell Carcinoma. neo 66, 261-270. doi:10.4149/neo 2018 180621n409

Zhen, Y., Fang, W., Zhao, M., Luo, R., Liu, Y., Fu, Q., et al. (2017). miR-374aCCND1-pPI3K/AKT-c-JUN Feedback Loop Modulated by PDCD4 Suppresses Cell Growth, Metastasis, and Sensitizes Nasopharyngeal Carcinoma to Cisplatin. Oncogene 36, 275-285. doi:10.1038/onc.2016.201

Zou, Y., Lin, X., Bu, J., Lin, Z., Chen, Y., Qiu, Y., et al. (2019). Timeless-Stimulated miR-5188-Foxo1/ $\beta$-Catenin-C-Jun Feedback Loop Promotes Stemness via Ubiquitination of $\beta$-Catenin in Breast Cancer. Mol. Ther. pii: S1525-0016 (19), 30398-30403.

Conflict of Interest: The reviewer YL declared a shared affiliation with one of the authors $\mathrm{YN}$ at time of review.

The remaining authors declare that the research was conducted in the absence of any commercial or financial relationships that could be construed as a potential conflict of interest.

Publisher's Note: All claims expressed in this article are solely those of the authors and do not necessarily represent those of their affiliated organizations, or those of the publisher, the editors and the reviewers. Any product that may be evaluated in this article, or claim that may be made by its manufacturer, is not guaranteed or endorsed by the publisher.

Copyright $\odot 2022 \mathrm{Liu}, \mathrm{Lv}$, Lin, Ning, Li, Liu and Chen. This is an open-access article distributed under the terms of the Creative Commons Attribution License (CC BY). The use, distribution or reproduction in other forums is permitted, provided the original author(s) and the copyright owner(s) are credited and that the original publication in this journal is cited, in accordance with accepted academic practice. No use, distribution or reproduction is permitted which does not comply with these terms. 